\title{
Spectral properties of the dynamic state transition in metal halide perovskite-based memristor exhibiting negative capacitance
}

Cite as: Appl. Phys. Lett. 118, 073501 (2021); https://doi.org/10.1063/5.0037916

Submitted: 17 November 2020 . Accepted: 30 January 2021 . Published Online: 16 February 2021

(iD) Cedric Gonzales, (D) Antonio Guerrero, and (iD) Juan Bisquert
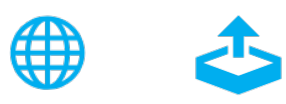

\section{ARTICLES YOU MAY BE INTERESTED IN}

Wavelength tunable single-mode lasing from cesium lead halide perovskite microwires

Applied Physics Letters 118, 071103 (2021); https://doi.org/10.1063/5.0035104

Free-standing and flexible graphene supercapacitors of high areal capacitance fabricated by laser holography reduction of graphene oxide

Applied Physics Letters 118, 071601 (2021); https://doi.org/10.1063/5.0038508

Impedance spectroscopy of perovskite/contact interface: Beneficial chemical reactivity effect The Journal of Chemical Physics 151, 124201 (2019); https://doi.org/10.1063/1.5111925

Challenge us.

What are your needs for periodic signal detection?

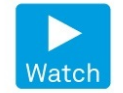

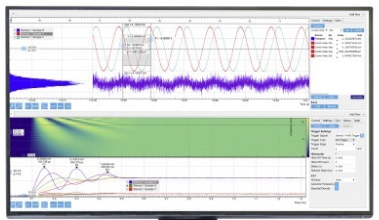

0
Zurich Instruments 


\title{
Spectral properties of the dynamic state transition in metal halide perovskite-based memristor exhibiting negative capacitance
}

Cite as: Appl. Phys. Lett. 118, 073501 (2021); doi: 10.1063/5.0037916

Submitted: 17 November 2020 - Accepted: 30 January 2021 •

Published Online: 16 February 2021

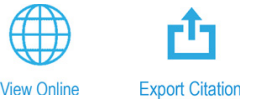

Cedric Gonzales, (D) Antonio Guerrero, ${ }^{\text {a) }}$ (D) and Juan Bisquert ${ }^{\text {a) }}$ (D)

\author{
AFFILIATIONS \\ Institute of Advanced Materials (INAM), Universitat Jaume I, 12006 Castelló, Spain \\ a) Authors to whom correspondence may be addressed: aguerrer@uji.es and bisquert@uji.es
}

\begin{abstract}
The evolution of device properties in memristor switching between high- and low-resistance states is critical for applications and is still highly subjected to significant ambiguity. Here, we present the dynamic state transition in a 2D Ruddlesden-Popper perovskite-based memristor device, measured via impedance spectroscopy. The spectral evolution of the transition exhibits a significant transformation of the low frequency arc to a negative capacitance arc, further decreasing the device resistance. The capacitance-frequency evolution of the device indicates that the appearance of the negative capacitance is intimately related to a slow kinetic phenomenon due to ionic migration and redistribution occurring at the perovskite/metal contact interface. In contrast, no negative capacitance arc is observed during the state transition of a memristor device where the contact is passivated by an undoped Spiro-OMeTAD interfacial layer. The switching mechanisms are entirely different, one due to interface transformation and the other due to filamentary formation.
\end{abstract}

Published under license by AIP Publishing. https://doi.org/10.1063/5.0037916

Resistive random access memory (ReRAM) devices have been gaining considerable interest due to their nonvolatile switching properties allowing in-memory computing analogous to the synaptic response in the human brain. ${ }^{1,2}$ The promising in-memory and neuromorphic computing capabilities of ReRAM devices aim to subvert the traditional Von Neumann architecture and overcome its limitations, i.e., heat wall and memory wall, signifying an eventual end to Moore's law. ${ }^{1,2}$ The resistive switching has been observed in oxide films in metal/oxide/metal structures, ${ }^{3-6}$ two-terminal organic semiconductors, ${ }^{7}$ silicon-based devices with complementary metal/oxide/semiconductor compatible architecture, ${ }^{8,9}$ and various halide perovskite materials. ${ }^{10-14}$ As the memristive response is demonstrated irrespective of the device design and configuration, the switching mechanism is still yet to be completely elucidated, making interpretations based on traditional models rather challenging.

Numerous interpretations have been proposed to explain the resistive switching mechanism specific to the device configuration. Most notably, the device switching mechanism in perovskite-based devices ranges from the formation and rupture of highly conducting filaments at applied voltage, ${ }^{10}$ nonfilamentary ionic and vacancy redistribution, ${ }^{11,15-17}$ to silver-iodide (AgI)-induced resistive switching. ${ }^{13}$ However, there has been no concrete consensus on the memristive switching mechanism. Several studies have conducted nondestructive impedance spectroscopy (IS) to qualitatively interpret the distinction between the memristor ON and OFF states., ${ }^{4,19}$ Despite the advances in memristive device designs, information on the device evolution during the dynamic state transition is still insufficient.

In this Letter, we demonstrate the evolution of the memristive switching in a two-dimensional (2D) Ruddlesden-Popper (RP) perovskite ReRAM device with particular focus on the transition state via IS. We have been able to obtain stable and reproducible IS results in these devices over a significant range of applied bias that enables a systematic investigation of the transition state from high to low resistance, providing insight into the electrical and physical origin of the transformation. The IS measurements reveal an evolution of the low frequency arc to a negative capacitance arc, suggesting an intimate correlation with the interfacial reactivity between perovskite and the metal contact layer. In contrast, an ReRAM device exhibiting stronger hysteresis with an interfacial layer between the perovskite and the metal contact did not feature a low-frequency negative capacitance arc.

The fabricated resistive random access memory (ReRAM) device was based on the $2 \mathrm{D} R \mathrm{RP}(\mathrm{PEA})_{2}(\mathrm{MA})_{\bar{n}-1} \mathrm{~Pb}_{\bar{n}} \mathrm{I}_{3 \bar{n}+1}$ perovskite (PEA $=$ phenylethylammonium and $\mathrm{MA}=$ methylammonium), where $\bar{n}=5$ is the dominant $n$ component. The $2 \mathrm{D}$ RP perovskite 
formulation properties, such as considerable ON/OFF ratio, good data retention capability, high environmental stability, good electrical conductivity, and reduced ion migration, were highly suitable for memory applications. ${ }^{11}$ The ReRAM device had a configuration of fluorinedoped tin oxide (FTO)/poly(3,4-ethylenedioxythiophene) polystyrene sulfonate (PEDOT:PSS)/2D RP perovskite/Ag $(15 \mathrm{~nm}) / \mathrm{Au}(85 \mathrm{~nm})$; herein, the sample is called the Ag ReRAM device. For comparison, an ReRAM device with a thin, undoped Spiro-OMeTAD interfacial layer in between the 2D perovskite and $\mathrm{Ag}$ was also fabricated (Spiro/Ag ReRAM). The Ag and Spiro/Ag ReRAM devices had effective active areas of 0.257 and $0.098 \mathrm{~cm}^{2}$, respectively. The relatively large device sizes were implemented for ease of fabrication and measurement. The dynamic transition state was investigated via a sequence of chronoamperometry (CA) for $5 \mathrm{~s}$ subsequently followed by impedance spectroscopy (IS) with a frequency range of $0.1 \mathrm{MHz}-0.1 \mathrm{~Hz}$ and an amplitude of $10 \mathrm{mV}$. The CA-IS sequence was conducted at varying voltages from $0 \mathrm{~V}$ to $2 \mathrm{~V}$ back to $0 \mathrm{~V}$ at $0.1 \mathrm{~V}$ increments. All measurements were carried out inside the glovebox filled with nitrogen under dark and controlled conditions using an Autolab PGSTAT-30 potentiostat.

The complex plane impedance plots of the IS spectra evolution for the Ag ReRAM device with the corresponding reconstructed current density-voltage $(j-V)$ curves from the CA measurements are shown in Figs. 1(a) and 1(c), respectively. The high-resistance state (HRS) or OFF state and low-resistance state (LRS) or ON state are highlighted accordingly, with the arrows indicating the scan direction. At $0 \mathrm{~V}$, the IS spectrum is in the initial OFF state exhibiting two distinct, well-defined impedance contours corresponding to the highand low-frequency arcs. The high frequency arc is generally attributed to the bulk response, while the low frequency arc is related to the perovskite/contact interface. ${ }^{20,21}$ At low applied voltages, up to $0.3 \mathrm{~V}$, the low-frequency resistance slightly increases with an observable noise usually connected to the interaction of ions with the contact. ${ }^{22}$ Conversely, the high-frequency resistance remains unchanged, indicating no significant difference in the bulk perovskite properties as the applied bias is modified. Approaching the threshold voltage, from $0.4 \mathrm{~V}$ to $0.5 \mathrm{~V}$, both the high- and low-frequency arcs begin to decrease correlated with modifications in both the bulk and the interfacial properties, respectively. Beyond the threshold voltage, in the transition state of $0.6 \mathrm{~V}$, the high-frequency arc begins to decrease more dramatically, suggesting a further transformation in the bulk. Most notably, the low-frequency arc begins to manifest as a negative capacitance arc. The negative capacitance arc persists up to $0.8 \mathrm{~V}$, just before the device

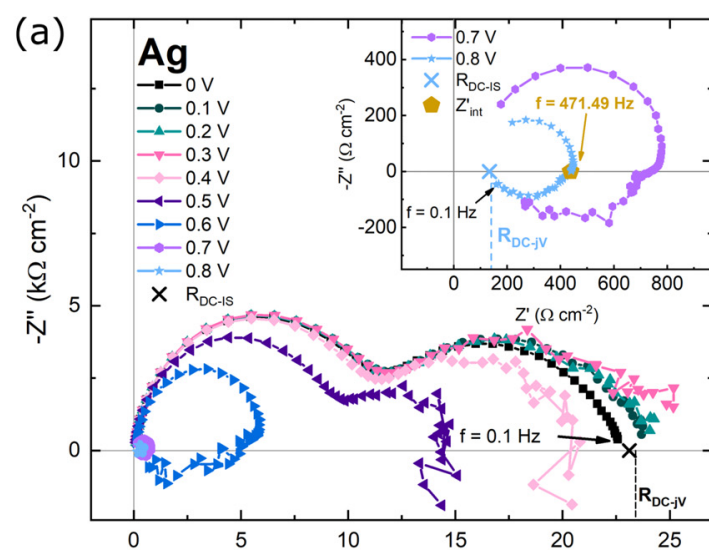

(c)

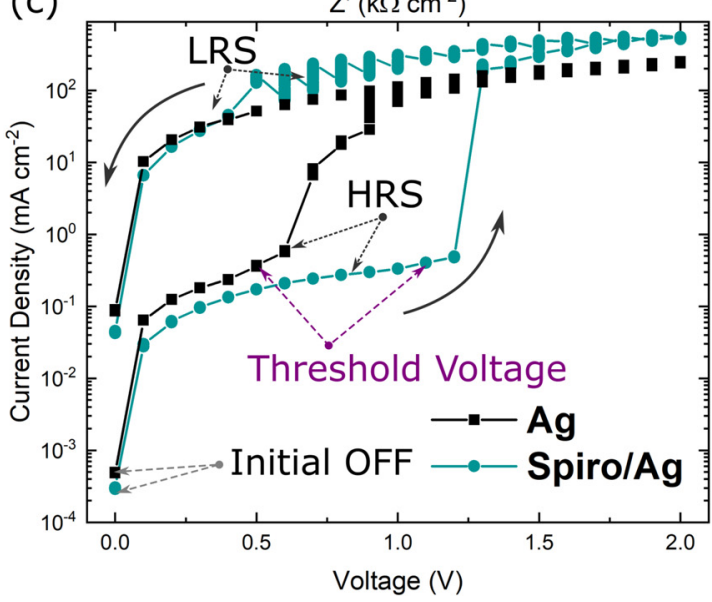

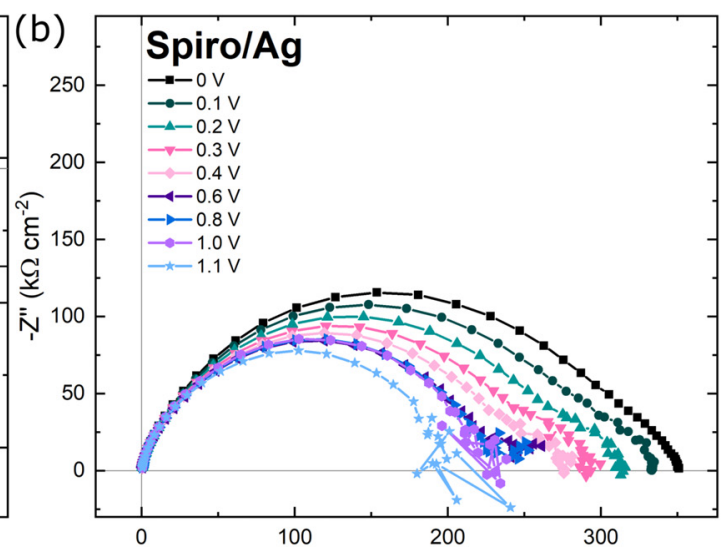

(d)

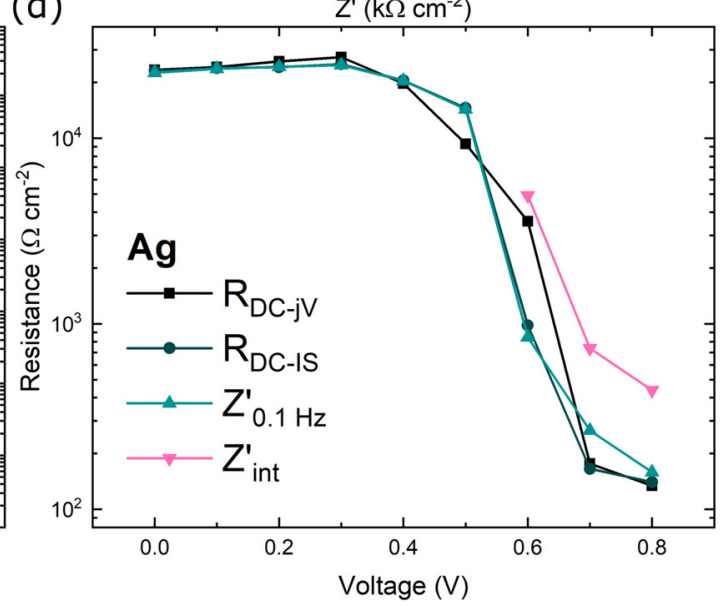

FIG. 1. (a) The IS spectra evolution of the Ag ReRAM device at representative voltages, with $R_{\mathrm{DC}-\mathrm{j} \mathrm{V}}, R_{\mathrm{DC}-\mathrm{IS}}$, and $\mathrm{Z}_{\mathrm{int}}^{\prime}$ highlighted for the representative OFF (OV) and transi-

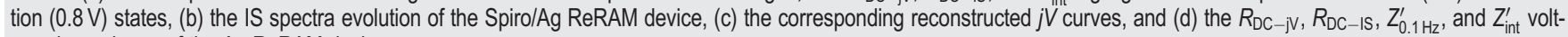
age dependence of the Ag ReRAM device. 
completely transitions to the $\mathrm{ON}$ state $(0.9 \mathrm{~V})$, exhibiting highly unstable IS spectra throughout the measurement frequency range.

The switching event of the Ag ReRAM device takes place within $3 \mathrm{~s}$ as revealed in CA measurements at the applied switching voltage of $V_{\text {app }}=0.9 \mathrm{~V}$ shown in Fig. 2(a). The current response of the Ag ReRAM device exhibits a continuous gradual increase in current density and then eventually stabilizes before $3 \mathrm{~s}$. We note that prior to each IS scan, a $5 \mathrm{~s}$ CA measurement is carried out. Hence, the observed reduction in resistances indicates a stabilized dynamic response in the bulk and perovskite/Ag interfacial properties. ${ }^{11,23}$ The gradual transition of the Ag ReRAM device implies a nonfilamentary switching mechanism due to ion migration and distribution at the perovskite/Ag interface. $^{11,15-17}$ The resistive switching mechanism has been attributed to the reversible reaction of formation and dissolution of the AgI monolayer at the 2D RP perovskite/Ag interface, sufficiently modifying the injection properties that control the electronic charge transfer rate. ${ }^{11}$

In order to obtain clearer insight into the origin of the negative capacitance arcs, the IS spectra evolution of the Spiro/Ag ReRAM device is investigated. The complex impedance plots of the IS spectra evolution for the Spiro/Ag ReRAM device at representative applied voltages are shown in Fig. 1(b). In contrast to the Ag ReRAM device, the initial OFF state of the Spiro/Ag ReRAM device exhibits two convoluted impedance contours with a significantly higher overall resistance. The higher overall resistance is expected for having the undoped Spiro-OMeTAD interfacial layer, due to its high transport resistance, inhibiting the direct contact of the $2 \mathrm{D}$ perovskite layer with the Ag metal contact. ${ }^{24}$ As the applied voltage is increased up to $0.4 \mathrm{~V}$, the overall resistance gradually decreases, suggesting a consistent modification in the Spiro-OMeTAD layer due to migrating ions' arrival at the interface. At higher applied voltages from $0.6 \mathrm{~V}$ to $1.0 \mathrm{~V}$, the IS spectra exhibit no significant change in overall resistance but have observable low frequency noise, suggesting an interaction of ions with the Ag contact similar to the Ag ReRAM device response at low applied voltages. This suggests that the undoped Spiro-OMeTAD layer acts as a physical barrier inhibiting the interaction between migrating ions and the Ag contact at lower applied voltages. Approaching the threshold voltage at $1.1 \mathrm{~V}$, the overall resistance appears to decrease once more with a more observable low-frequency noise, indicating a further change in the device properties and an increased interaction of ions with the Ag contact.

The higher threshold voltage is due to the high transport resistance of the Spiro-OMeTAD layer necessitating a higher applied bias to compensate the voltage drop experienced by the device. The
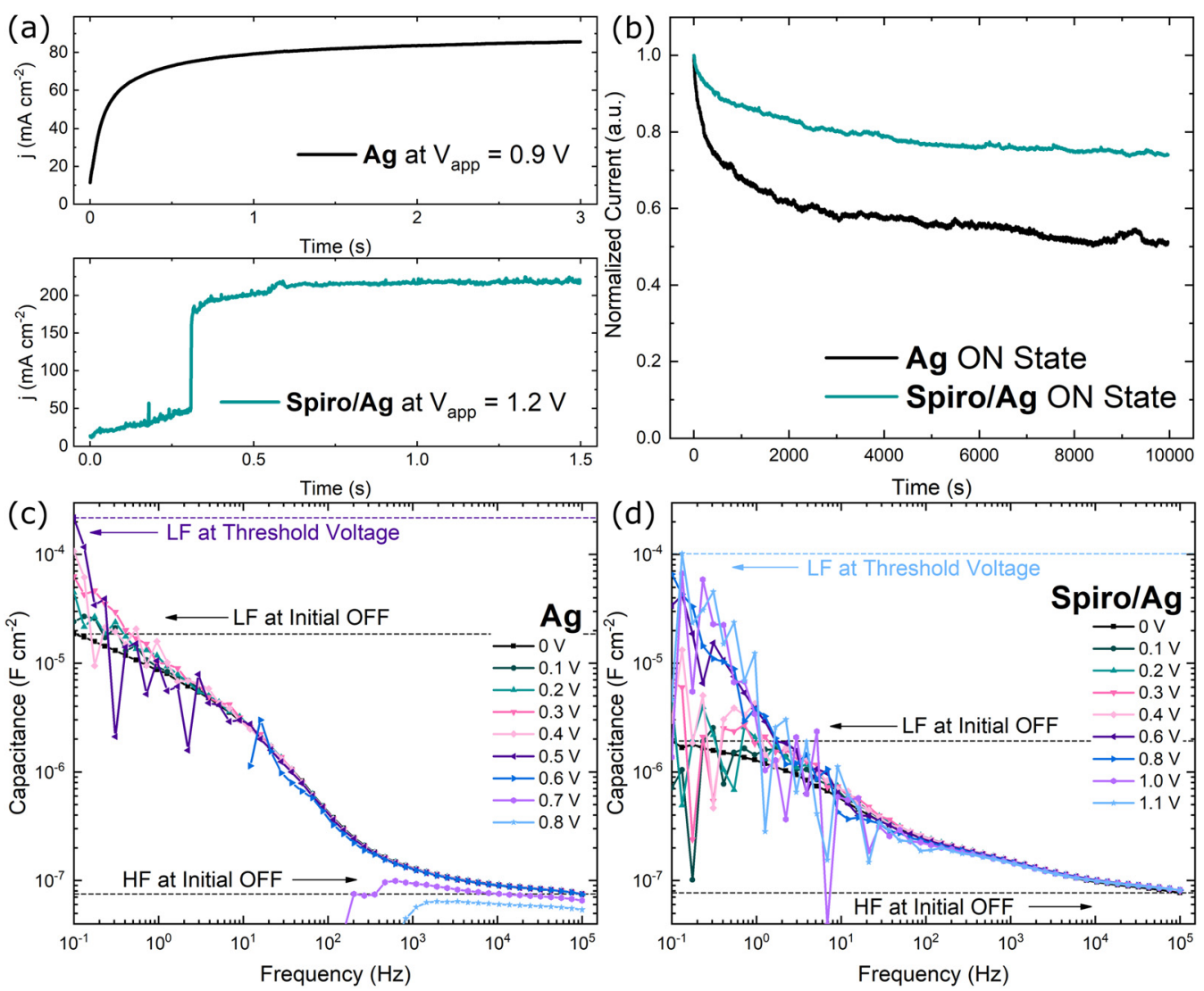

FIG. 2. (a) The chronoamperometry measurements monitoring the switching speed for the $\mathrm{Ag}$ and Spiro/Ag ReRAM devices at switching voltages ( $V_{\text {app }}$ ) of $0.9 \mathrm{~V}$ and $1.2 \mathrm{~V}$, respectively, (b) the ON state retention measurement of the Ag and Spiro/Ag ReRAM devices measured at $V_{\text {read }}=0.3 \mathrm{~V}$, and the C-f plots of the (c) Ag and (d) Spiro/Ag ReRAM devices at varying applied voltages, highlighting the high- and low-frequency capacitances in the initial off state and at the threshold voltage. 
Spiro/Ag ReRAM device then abruptly switches to the ON state at $1.2 \mathrm{~V}$, without the low-frequency negative capacitance arc, as opposed to the Ag ReRAM device with a more gradual transition state from $0.6 \mathrm{~V}$ to $0.8 \mathrm{~V}$. Correspondingly, an abrupt switching at $0.3 \mathrm{~s}$ of the Spiro/Ag ReRAM device is observed at $V_{\text {app }}=1.2 \mathrm{~V}$ and then continues to increase in current density until it eventually stabilizes before $1.5 \mathrm{~s}$ as shown in Fig. 2(a). The abrupt transition of the Spiro/Ag ReRAM device suggests the formation of conductive filaments within the Spiro-OMeTAD layer. ${ }^{10,12,13,17}$ Moreover, both devices exhibit high $\mathrm{ON}$ state retention times of $>10^{4} \mathrm{~s}$ at a read voltage of $0.3 \mathrm{~V}$ as shown in Fig. 2(b). Notably, the Ag and the Spiro/Ag ReRAM ON state currents gradually decrease and stabilize at $\sim 51 \%$ and $\sim 74 \%$ of the initial value, respectively. The larger sustained ON state current of the Spiro/Ag ReRAM device further implies a filamentary switching mechanism. ${ }^{25}$ The higher overall resistance due to the undoped Spiro$\mathrm{OMeTAD}$ layer together with the filamentary resistive switching results in a higher ON/OFF ratio of $\sim 3$ orders of magnitude for the Spiro/Ag ReRAM device as compared to $\sim 2$ orders of magnitude for the Ag ReRAM device. The absence of the low-frequency negative capacitance arcs in the Spiro/Ag ReRAM resistive switching indicates that the negative capacitance is intimately correlated with the ion migration and redistribution, promoting the reactivity at the perovskite/Ag interface.

In order to validate the low-frequency negative capacitance arcs of the Ag ReRAM device, the differential direct current (DC) resistance obtained from the reconstructed $j V$ curves $\left(R_{\mathrm{DC}-\mathrm{jV}}\right)$ is compared with DC resistance of the IS spectra $\left(R_{\mathrm{DC}-\mathrm{IS}}\right) . R_{\mathrm{DC}-\mathrm{jV}}$, defined as the resistance at stationary conditions $(f=0 \mathrm{~Hz})$, obeys the relationship given by $R_{\mathrm{DC}-\mathrm{jV}}=\partial V / \partial I$. From the complex impedance plot, $R_{\mathrm{DC}-\mathrm{IS}}$ is obtained from the real impedance intercept of the IS extrapolated at $f \rightarrow 0 \mathrm{~Hz}$. $R_{\mathrm{DC}-\mathrm{jV}}$ and $R_{\mathrm{DC}-\mathrm{IS}}$ plotted against the IS spectra at representative OFF state $(0 \mathrm{~V})$ and transition state $(0.8 \mathrm{~V})$ voltages are illustrated in Fig. 1(a) and the inset of Fig. 1(a), respectively. At the OFF state voltage, $R_{\mathrm{DC}-\mathrm{jV}}$ is in good agreement with $R_{\mathrm{DC}-\mathrm{IS}}$, confirming a high correlation between the reconstructed $j V$ curve and the measured IS spectra. Consequently, the real impedance at the lowest measurement frequency $\left(Z_{0.1 \mathrm{~Hz}}^{\prime}\right)$ corresponds well to both $R_{\mathrm{DC}}$ values. On the other hand, the real impedance intercept $\left(Z_{\text {int }}^{\prime}\right)$ occurs at an intermediate frequency for the IS spectrum at $0.8 \mathrm{~V}$, exhibiting the negative capacitance arc, highlighted in the inset of Fig. 1(a). The correlation among $R_{\mathrm{DC}-\mathrm{jV}}, R_{\mathrm{DC}-\mathrm{IS}}, Z_{0.1 \mathrm{~Hz}}^{\prime}$, and $Z_{\text {int }}^{\prime}$ is plotted in Fig. $1(\mathrm{~d})$. The correlation among the resistances shows that the negative capacitance arc provides a major contribution to the DC resistance of the device, which highlights the significance of the negative capacitance phenomenon for practical device operation. In addition, it has been frequently reported that slight variations in steady-state conditions of the system under measurement can significantly affect the device response during data collection, especially at low frequencies requiring longer measurement times. ${ }^{11,26}$ However, in our case, the measurement is reproducible, indicating no drift of the physical system and, thus, obtaining a reliable spectral response. It is noted that the current measurement for the reconstructed $j V$ curve is conducted before the IS, suggesting that the high correlation among $R_{\mathrm{DC}-\mathrm{jV}}, R_{\mathrm{DC}-\mathrm{IS}}$, and $Z_{0.1 \mathrm{~Hz}}^{\prime}$ is indicative of a stable IS response by the ReRAM device for voltages prior to the ON state. The further reduction in resistance manifested by the lowfrequency negative arcs indicates a slow kinetic interaction at the perovskite/Ag interface occurring during the dynamic state transition.
Investigating the evolution of the capacitance-frequency $(C-f)$ plots provides further information on the charge distribution during state transitions of the ReRAM devices. The $C-f$ plots of the Ag and Spiro/Ag ReRAM devices are shown in Figs. 2(c) and 2(d), respectively. The high-frequency capacitive plateau is generally attributed to the bulk capacitance of the perovskite layer, while the low frequency plateau is attributed to electrode polarization due to charge accumulation. ${ }^{20,23,27}$ Both devices exhibit nearly identical high-frequency capacitance in the initial OFF state, confirming that the 2D RP perovskite layers are of the same bulk capacitive properties. However, the OFF state low frequency capacitance of the Spiro/Ag ReRAM device features a lower capacitance than the Ag ReRAM device. The lower low-frequency capacitance of the OFF state is attributed to the SpiroOMeTAD layer acting as a physical barrier between the perovskite layer the Ag metal contact. The decrease in capacitance is consistent with the typical parallel plate capacitor governed by $C=\varepsilon \varepsilon_{0} / d$, where $C$ is the capacitance per unit area, $\varepsilon_{0}$ is the vacuum permittivity, and $d$ is the separation between the plates. As the voltage is increased for the Ag ReRAM device, the low-frequency capacitance gradually increases up to $\sim 10^{-4} \mathrm{~F} \mathrm{~cm}^{-2}$ at the threshold voltage, subsequently followed by a dramatic drop at the transition state voltages. Markedly, the lowfrequency capacitances of the Spiro/Ag ReRAM device at the threshold voltage approach the low-frequency capacitance of the Ag ReRAM device at the threshold voltage. The high correspondence of lowfrequency capacitances at the threshold voltage suggests a similar device response consistent with the IS spectra evolution. The delayed capacitive response of the Spiro/ReRAM device upholds the initial impediment to the interfacial reactivity of migrating ions with the Ag metal contact by the thin, undoped Spiro-OMeTAD layer, necessitating a higher applied voltage to promote the chemical reactivity.

In summary, we have demonstrated the dynamic state transitions in $2 \mathrm{D} \mathrm{RP}$ perovskite-based memristor devices via IS. The interfacial reactivity between the perovskite and the Ag metal contact resulted in a gradual state transition, indicative of a nonfilamentary switching mechanism, wherein the distinct, well-defined low-frequency arc transformed into a persistent negative capacitance arc during the device transition state. In contrast, a thin, undoped Spiro-OMeTAD interfacial layer, acting as a physical barrier with a higher overall resistance, impeded the reactivity between the migrating ions and the metal contact. Consequently, the ReRAM device with the Spiro-OMeTAD interfacial layer exhibited a higher ON/OFF ratio with an abrupt transition from the OFF state to the ON state, suggesting the filament formation within the Spiro-OMeTAD layer, but did not feature the negative capacitance arc. The switching and retention measurements further substantiate the distinction between the resistive switching mechanisms. The high correlation between the differential DC resistance from the reconstructed $j V$ curve with the extrapolated DC resistance from the IS spectra suggested a further reduction in device resistance, corroborating the validity of the negative capacitance arcs. The $C-f$ evolution further confirmed the impediment to ionic migration and reactivity by the Spiro-OMeTAD interfacial layer. The dynamic IS spectra and $C-f$ evolution for both devices indicated an intimate correlation between the low frequency negative capacitance and the interfacial interaction attributed to a slow ionic migration and redistribution, leading to the formation and dissolution of AgI. Despite the relatively large device sizes, memristive responses were observed for both devices. Down-scaling the device dimensions for 
practical applications is expected to exhibit significantly stronger memristive behavior on the nanometer scale. ${ }^{28}$ The insight into the dynamic state transition investigated by IS would allow for ReRAM device designs with tailored switching properties in terms of the ON/ OFF ratio, threshold voltage, and transition state control.

We thank the financial support from Generalitat Valenciana for a Prometeo (No. PROMETEU/2020/028), Grisolia Grant (No. GRISOLIAP/2019/048), and Ministerio de Ciencia y Innovación (No. PID2019-107348GB-100).

\section{DATA AVAILABILITY}

The data that support the findings of this study are available from the corresponding author upon reasonable request.

\section{REFERENCES}

${ }^{7}$ D. Ielmini and H. S. P. Wong, Nat. Electron. 1, 333-343 (2018).

${ }^{2}$ M. A. Zidan, J. P. Strachan, and W. D. Lu, Nat. Electron. 1, 22-29 (2018).

${ }^{3}$ A. Beck, J. G. Bednorz, C. Gerber, C. Rossel, and D. Widmer, Appl. Phys. Lett. 77(1), 139-141 (2000).

${ }^{4}$ C. S. Dash, S. Sahoo, and S. R. S. Prabaharan, Solid State Ionics 324(1), 218-225 (2018).

${ }^{5}$ M. H. Lee, K. M. Kim, G. H. Kim, J. Y. Seok, S. J. Song, J. H. Yoon, and C. S. Hwang, Appl. Phys. Lett. 96(15), 152909 (2010).

${ }^{6}$ S. Seo, M. J. Lee, D. C. Kim, S. E. Ahn, B. H. Park, Y. S. Kim, I. K. Yoo, I. S. Byun, I. R. Hwang, S. H. Kim, J. S. Kim, J. S. Choi, J. H. Lee, S. H. Jeon, S. H. Hong, and B. H. Park, Appl. Phys. Lett. 87(26), 263507 (2005).

${ }^{7}$ F. Verbakel, S. C. J. Meskers, R. A. J. Janssen, H. L. Gomes, M. Cölle, M. Büchel, and D. M. De Leeuw, Appl. Phys. Lett. 91(19), 192103-192104 (2007).

${ }^{8}$ S. H. Jo, T. Chang, I. Ebong, B. B. Bhadviya, P. Mazumder, and W. Lu, Nano Lett. 10(4), 1297-1301 (2010).

${ }^{9}$ S. Kim, H. Kim, S. Hwang, M. H. Kim, Y. F. Chang, and B. G. Park, ACS Appl. Mater. Interfaces 9(46), 40420-40427 (2017).
${ }^{10}$ W. Tress, J. Phys. Chem. Lett. 8(13), 3106-3114 (2017).

${ }^{11}$ A. Solanki, A. Guerrero, Q. Zhang, J. Bisquert, and T. C. Sum, J. Phys. Chem. Lett. 11(2), 463-470 (2020).

${ }^{12}$ Y. He, G. Ma, X. Zhou, H. Cai, C. Liu, J. Zhang, and H. Wang, Org. Electron. 68, 230-235 (2019).

${ }^{13}$ S. Ge, Y. Huang, X. Chen, X. Zhang, Z. Xiang, R. Zhang, W. Li, and Y. Cui, Adv. Mater. Interfaces 6(7), 1802071-1802079 (2019).

${ }^{14}$ P. C. Harikesh, B. Febriansyah, R. A. John, and N. Mathews, MRS Bull. 45(8), 641-648 (2020).

${ }^{15}$ C. Gu and J. S. Lee, ACS Nano 10(5), 5413-5418 (2016).

${ }^{16}$ X. Guan, W. Hu, M. A. Haque, N. Wei, Z. Liu, A. Chen, and T. Wu, Adv. Funct. Mater. 28(3), 1704665 (2018).

${ }^{17}$ J. Zhu, T. Zhang, Y. Yang, and R. Huang, Appl. Phys. Rev. 7(1), 011312 (2020).

${ }^{18}$ Y. H. You, B. S. So, J. H. Hwang, W. Cho, S. S. Lee, T. M. Chung, C. G. Kim, and K. S. An, Appl. Phys. Lett. 89(22), 222105 (2006).

${ }^{19}$ R. Schmitt, M. Kubicek, E. Sediva, M. Trassin, M. C. Weber, A. Rossi, H. Hutter, J. Kreisel, M. Fiebig, and J. L. M. Rupp, Adv. Funct. Mater. 29(5), 1804782 (2019).

${ }^{20}$ A. Guerrero, G. Garcia-Belmonte, I. Mora-Sero, J. Bisquert, Y. S. Kang, T. J. Jacobsson, J. P. Correa-Baena, and A. Hagfeldt, J. Phys. Chem. C 120(15), 8023-8032 (2016).

${ }^{21}$ W. Peng, C. Aranda, O. M. Bakr, G. Garcia-Belmonte, J. Bisquert, and A. Guerrero, ACS Energy Lett. 3(7), 1477-1481 (2018).

${ }^{22}$ C. Li, A. Guerrero, S. Huettner, and J. Bisquert, Nat. Commun. 9(1), 1 (2018).

${ }^{23}$ C. Aranda, J. Bisquert, and A. Guerrero, J. Chem. Phys. 151(12), 124201 (2019).

${ }^{24}$ F. Fabregat-Santiago, J. Bisquert, E. Palomares, S. A. Haque, and J. R. Durrant, J. Appl. Phys. 100(3), 034510 (2006).

${ }^{25}$ F. Alibart, E. Zamanidoost, and D. B. Strukov, Nat. Commun. 4, 1-7 (2013).

${ }^{26}$ S. Ravishankar, M. García-Batlle, J. Bisquert, G. Garcia-Belmonte, J. Odrobina, and C.-A. Schiller, J. Phys. Chem. C 124(29), 15793 (2020).

${ }^{27}$ O. Almora, I. Zarazua, E. Mas-Marza, I. Mora-Sero, J. Bisquert, and G. GarciaBelmonte, J. Phys. Chem. Lett. 6(9), 1645-1652 (2015).

${ }^{28}$ D. B. Strukov, G. S. Snider, D. R. Stewart, and R. S. Williams, Nature 453(7191), 80-83 (2008). 